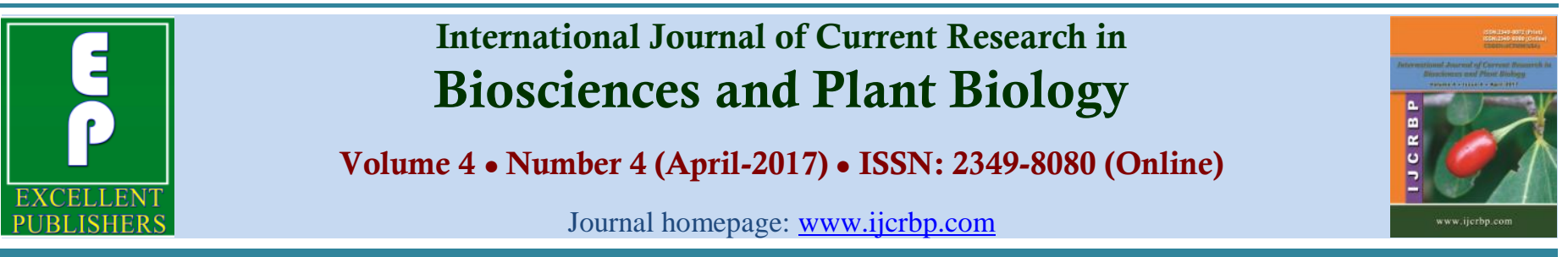

\title{
The Periodicity of Light and the Nature of Chlorophyll
}

\author{
Zhe Yin* \\ Department of Mathematics, Yanbian University, China \\ *Corresponding author.
}

\begin{tabular}{|c|c|}
\hline \multirow{6}{*}{$\begin{array}{l}\text { Abstract } \\
\text { In this paper, the gravitational wave theory is used to clarify the propagation of the } \\
\text { gravitational waves in the intertwined form of electrons. The light has periodic properties, } \\
\text { and the light has the same form of block entanglement. The degree of entanglement is } \\
\text { expressed by relative energy. Each photoperiod contains four sub-process morphologies, } \\
\text { each sub-process morphology, forming a separate, electron-group and entangled form of } \\
\text { relative energy set which are all determine the nature of chlorophyll. }\end{array}$} & Article Info \\
\hline & $\begin{array}{l}\text { Accepted: } 27 \text { March } 2017 \\
\text { Available Online: } 06 \text { April } 2017\end{array}$ \\
\hline & Keywords \\
\hline & Chlorophyll \\
\hline & Gravitational wave \\
\hline & Light \\
\hline
\end{tabular}

\section{Introduction}

It has been proposed in 2010 that substances change to the direction of increase of atomic weight and molecular weight (Yin, 2010) and it has also been proposed that spiral trajectory of gravitational waves (Weber, J., 1959 and 2016) and the variable force field is the condition of energy conversion in February 2016 (Yin and Liu, 2016a,b). The motion trajectory of the electrons, proposed the law of motion that, spiral trajectory gravitational wave produces entanglement in the process of convergence and divergence, increases the energy, in the trajectory of Mobius', and spiral gravitational wave convergences inwards in March 2016 (Yin, 2016; Yin, Liu, 2016a,b).

Inward convergence is equivalent that the centripetal force is greater than buoyancy and other external field forces, outward divergence equivalent buoyancy and other external field force is greater than the centripetal force.
The following description provides the use of gravitational wave theory, to elucidate the propagation pattern of light between the electrons of the gravitational waves. The light has the entanglement between the periodic and the optical blocks with the same properties. The degree of entanglement between the light blocks is expressed by relative energy. Each photoperiod contains four sub-process morphologies, each sub-process morphology, forming a separate, electron-group and entangled form of relative energy set. Determine the nature of chlorophyll.

\section{Theoretical preparation}

Theorem 1 (Gravitational waves existence theory) (Yin et al., 2016; Yin, 2017a, b, c).

There are A, B two points. A is the wave source of gravitational field, and $\mathrm{B}$ is a point in the gravitational 
field. The existence of energy rotational motion (including proton, neutron, atomic nucleus or planet) at $A$ is the necessary and sufficient condition of the existence of gravitational waves at B. Direction is the bidirection of the path tangent at point $\mathrm{B}$, and the limit of the convergence direction is A point.

Theorem 2 (Gravitational wave stability theory) (Yin et al., 2016; Yin, 2017a, b, c)

A is a gravitational wave source, $\mathrm{B}$ is a point in gravitational field of $\mathrm{A}$. The necessary and sufficient conditions of stable gravitational wave at B point are:

$$
\frac{d r}{d \theta}=b \quad,(\mathrm{~b} \text { is a constant })
$$

Inference of theorem 2: If a gravitational wave track meet Archimedean spiral, this must be stable gravitational waves.

There is, $r=a+b \theta$.

$\mathrm{r}$ is the distance of A to $\mathrm{B}$; $\mathrm{a}$ is the spiral length of $\mathrm{A}$ to $\mathrm{B} ; \mathrm{b}$ is the distance between the spirals.

\section{Photoperiodicity and Chlorophyll theorem}

According to the gravitational wave equation, the periodic law of light is obtained. Gravitational waves are the propagation of light. Light is the same form of electronic, entangled particles in the form of transmission. Photons have relative energy and quality. Through the electronic entanglement, supplement and increase the energy, gradually increase the wavelength.

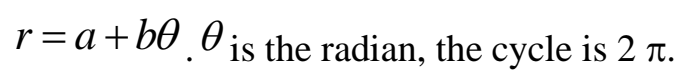

Theorem 1: The wave of light is periodic and its period is $2 \pi$

According to the independence, symmetry, monotonic principle, light has only four forms. (Four sub-process morphologies of the photoperiod).

\section{Theorem 2: Four independent morphological equations of light}

The first sub-process morph equation:

$$
r=a+b(2 k \pi+0), k=0,1,2,3, \ldots
$$

The second sub-process morph equation:

$$
r=a+b\left(2 k \pi+\frac{\pi}{2}\right), k=0,1,2,3, \ldots
$$

The third sub-process morph equation:

$$
r=a+b(2 k \pi+\pi), k=0,1,2,3, \ldots
$$

The fourth sub-process morph equation:

$$
r=a+b\left(2 k \pi+\frac{3 \pi}{2}\right), k=0,1,2,3, \ldots
$$

As in different environments, hydrogen atoms can be positively charged or negatively charged, the electron entangled state (photon) can be positively charged or negatively charged. Each sub-process of electrons forms a separate set of photonics form and share relative energy.

Anywhere on earth, four kinds of photon clusters coexist. The relative energy of the photon is proportional to the wavelength.

Theorem 3: Point on the earth, with a certain relative energy, four kinds of photon collection, the wavelength range is:

The wavelength range of the first sub-process form: [380, 495], unit $\mathbf{n m}$;

The Wavelength range of the second sub-process form: [476, 620], unit nm;

The wavelength range of the third sub-process form: [570, 750], unit nm;

The wavelength range of the fourth sub-process form: [590, 790] unit $\mathrm{nm}$.

Visible light in the wavelength range [380,450] shows purple, Because of the periodic reason, there is an aggregation state of energy between blue (first interval) and red (fourth interval). The relative energy represents the degree of entanglement and the total amount of photons, and the color represents the density of the photon. Density determines the color of any substance. The density is proportional to the reflectivity of light.

Photosynthesis is the process of converting light energy into chemical energy. Chlorophyll absorbs energy from light and converts carbon dioxide into carbon. Chlorophyll a, b, accept the light energy range is 
different. Chlorophyll c, d, e are different forms in different media. Chlorophyll is acceptable, the energy of electronically entangled particles is much smaller than that of hydrogen ions, so it does not participate in hydrogen transfer and hydrogen oxidation and reduction. Only electronically transmitted and conjugated.

\section{Theorem 4 (chlorophyll nature):}

The first sub-process and the fourth sub-process are the monotonically increasing intervals of the equation, so chlorophyll only has these two processes to accept energy.

The quality and energy of the four sub-process photons are different. Determine the specific role of the four bases of biological DNA.

\section{Conclusion}

In this paper, the use of gravitational wave properties to clarify the light entanglement and relative energy formation process and to clarify the principle of the four forms of light is explained. It is clarified that chlorophyll only accepts energy in the first sub-process and the fourth sub-process.

\section{Conflict of interest statement}

Author declares that there is no conflict of interest.

\section{References}

Weber, J., 1959, 2016. Gravitational Wave, Laser Interferometer Gravitational Wave Observatory. 2016.2.11.23, 30 .

Yin, Z., 2010. The co-planarity and symmetry principle of earthquake occurrence. Int. J. Geosci. 1(1), 3843.

Yin, Z., 2016. Zhe Yin's energy and wave theorem. Int. J. Engg. Adv. Res. Technol. 2(3), 44-45.

Yin, Z., 2017a. The function and relation of proton and neutron. Int. J. Recent Sci. Res. 8(1), 15321-15324.

Yin, Z., 2017b. Effect of Gravitational Wave on DNA Base. Int. J. Recent Sci. Res. 8(2), 15705-15707.

Yin, Z., 2017c. The formation of elements. IOSR J. Appl. Phys. 9(2), 08-13.

Yin, Z., Liu, D., 2016a. Electron trajectory. Int. J. Appl. Sci. Math. 3( 2), 56-57.

Yin, Z., Liu, D., 2016b. Causes of sound wave on the moon. Int. J. Eng. Appl. Sci. 3(2), 56-58.

Yin, Z., Zhang, W., Bie, H., 2016. Zhe Yin's theorem of molecular biology. Acad. J. Sci. Res. 4(4), 99-102.

\section{How to cite this article:}

ZIn,Y., 2017. The periodicity of light and the nature of chlorophyll. J. Curr. Res. Biosci. Plant Biol. 4(4), 46-48. doi: https://doi.org/10.20546/ijcrbp.2017.404.008 